\title{
A Comprehensive Review: Prevention and Control of SARS Co-V-2
}

\section{Pramod Chauhan ${ }^{1}$, Pratima Katiyar ${ }^{2 *}$, Kalpana Kushwaha ${ }^{2}$, Shivam Kumar Verma ${ }^{1}$, Amana Parveen ${ }^{1}$ and Raghvendra Kumar Yadav ${ }^{1}$}

${ }^{1}$ Research Scholar, School of Pharmaceutical Sciences, CSJM University, Kanpur, U.P. India

${ }^{2}$ Assistant Professor, School of Pharmaceutical Sciences, CSJM University, Kanpur, U.P. India

*Corresponding Author: Pratima Katiyar, Assistant Professor, School of

Pharmaceutical Sciences, CSJM University, Kanpur, U.P. India.
Received: October 14, 2021

Published: December 16, 2021

(C) All rights are reserved by Pratima Katiyar., et al.

\begin{abstract}
Background: Millions of people in the world have been infected by respiratory viral infections (RVIs) especially upper respiratory infections such as pneumonia and deeper pulmonary infections. About 200 recognized respiratory viruses exist on the planet. They are broadly classified into a family of DNA viruses (Adenoviridae) and RNA viruses (Orthomyxoviridae, Paramyxoviridae, Picornaviridae, and Coronaviride). Among them, SARS-CoV-2 develops a disease named COVID-19. A big cause of the pandemic situation since 2019 and have a considerable impact on human life. Therefore, the prevention and control of SARS Co-V2 are major aims for researchers. Main Body: The lack of effective vaccination against acute respiratory syndrome coronavirus-2 [SARS-CoV-2] infection has prompted increased efforts in direction of new medicines search. The repurposing of existing drugs has brought great hope for the treatment of pandemic covid-19 such as Ivermectin, an FDA-approved broad-spectrum antiparasitic recently, demonstrated significant antiviral activity in vitro against SARS-CoV-2. Adamantane derivatives have been also reported as antiviral drugs for respiratory viral infections. These drugs are reused in SARS CoV-2 treatment in the early stages for prophylactic purposes. Various stages are involved in virus propagation in a host cell, termed as "virus life cycle". So, all stages in the virus life cycle are imminent targets for the discovery of new antiviral entities.
\end{abstract}

Conclusion: This review paper summarized all aspects of medicinal approaches and biological approaches to control and management of covid infection.

Keywords: Respiratory Viral Infections; SARS Co-v-2; Respiratory Syndrome; Adamantine; Antiviral Drugs; Virus Life Cycle; Vaccine

\section{Introduction}

Respiratory tract infections are the most common infection caused by pathogenic viruses [1]. Upper respiratory tract infections are mostly caused by adenoviruses such as pharyngitis, tonsilitis. Mostly two category viruses are rhinoviruses (common cold virus influenza virus $80 \%$ ) and coronavirus (20\%) [2]. These viruses can cause various respiratory disorders such as acute bronchitis, COPD (Chronic obstructive pulmonary disease), asthma, systemic fibrosis, and pneumonia. Life-threatening RVIs are sometime appears in unhealthy people and immunocompromised persons and maybe a healthy person associated with other infections.

A coronavirus has been belonging to the coronoviridae family, emerged in 2019 and now it is a worldwide pandemic disease declared by WHO. The SARS-CoV-2 disease is characterized by various respiratory distress-related symptoms such as strain in breathing, eating, coughing, fever, and severe cases of diarrhea [3]. The coronavirus is spread out over humans and shows high interspecies flexibility. Corona Virus has low pathogenicity and is linked to 
respiratory and gastrointestinal disorders that are usually mild to moderate in severity. The SARS-CoV- 2 seems to depress the immune system of the body leading to cytokine storms and gradually harm to other organs of patients [4]. So, far in absence of effective therapy, some precautional instructions are given by WHO such as social distancing, hand hygiene, and personal protection for people [5]. As a result, finding different therapeutic ways to prevent COVID-19 is a major priority. Therefore, there is a high priority requirement to identify various therapeutic approaches for the prevention of COVID-19 [6].

\section{Viruses}

Viruses are obligatory intracellular parasites. Viruses must gain access to target cells and take over the host cellular machinery to spread and make progeny viruses [7]. The several stages involved in virus propagation inside cells are referred to as the "viral life cycle." The three stages of the virus's life cycle are entry, genome replication, and exit. Researchers are focus on entry and escape, where virus mechanisms share a lot of similarities [8]. Genome replication, on the other hand, is covered in Parts II to IV (Part II Part III Part III-1Part III-2Part IV), in which viral families are discussed individually [9]. Viruses do not have all the enzymes necessary for viral replication. A virus, on the other hand, can take over cellular machinery and use it to create new viral particles within the host cell. Because prokaryotic organisms lack a nucleus and organelles, bacteriophages can only replicate in the cytoplasm. Except for large DNA viruses such as poxviruses, which may replicate in the cytoplasm, most DNA viruses can multiply in the nucleus of eukaryotic cells. RNA viruses that are infectious replicate frequently in the cytoplasm of animal cells.

\section{Stages of virus life cycle}

\section{Viral entry}

The recognition of viral receptors by a virus particle is the first step in virus infection. Attachment, penetration, cytoplasmic trafficking, and uncoating are the four steps of viral entry. Because these processes are frequently linked to one another, the split into four steps is ambiguous, but it suits the goal of exposition [10].

\section{Attachments}

The first meeting of virus particles with host cells, which involves two types of host proteins on the plasma membrane, is referred to as the attachment.
- Attachment factors

- Viral receptors.

The attachment factor on the cell surface attracts and holds virus particles, making it easier for the viral particle to engage with the entrance receptor. Glycosaminoglycans like heparins serve as attachment factors for a variety of viruses, indicating the attachment factors' broader specificity [11].

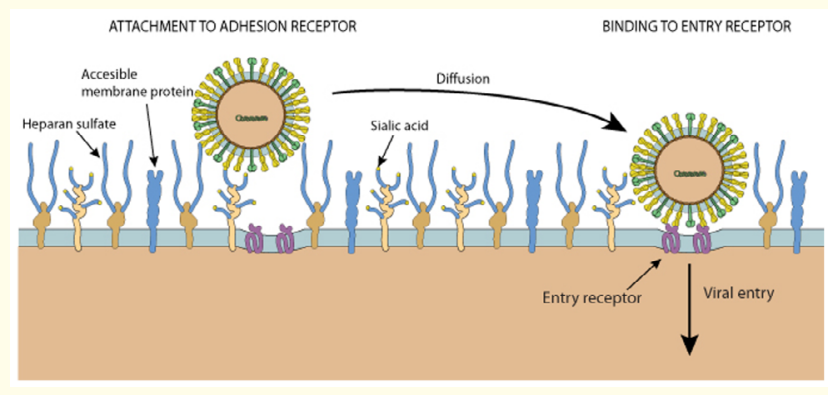

Figure 1: Virus attachment of host cell.

\section{Penetration}

The viral particle has been attached to the target cells the next stage is for it to penetrate the cytoplasm. Whether the penetration is enclosed or not, the method is different. Direct fusion or receptor-mediated endocytosis is the two ways employed to endocytose encapsulated viruses [12].

The method of penetration for nonenveloped naked viruses is receptor-mediated endocytosis.

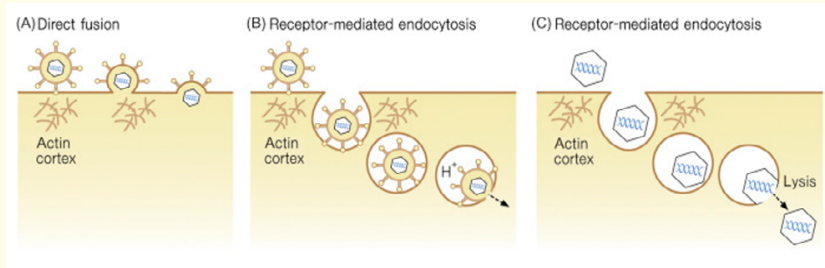

Figure 2: There are three types of viral penetration.

\section{Uncoating}

The viral genome becomes exposed to cellular machinery for viral gene expression as the virus particles approach the site of repli-

Citation: Pratima Katiyar., et al. "A Comprehensive Review: Prevention and Control of SARS Co-V-2". Acta Scientific Pharmaceutical Sciences 6.1 (2022): 
cation, from the cell periphery to the perinuclear region, a process known as uncoating. Uncoating is frequently associated with endocytic or cytoplasmic trafficking [13].
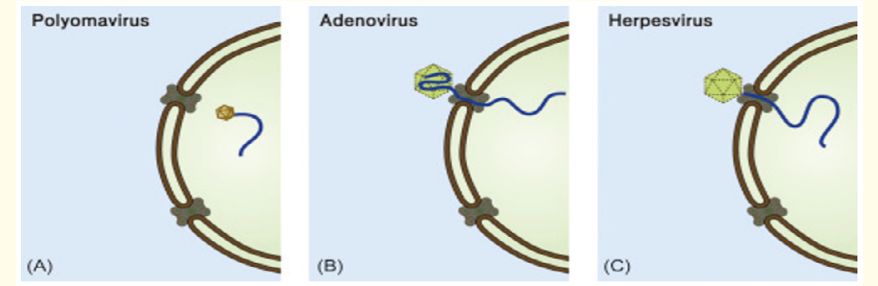

Figure 3: Strategies for the nuclear entry of DNA viruses.

\section{Release}

The virus particles are released when infected cells are lysed. Because the cell membrane that contains the formed virus particles is deconstructed, no specific exit mechanism is required [14].

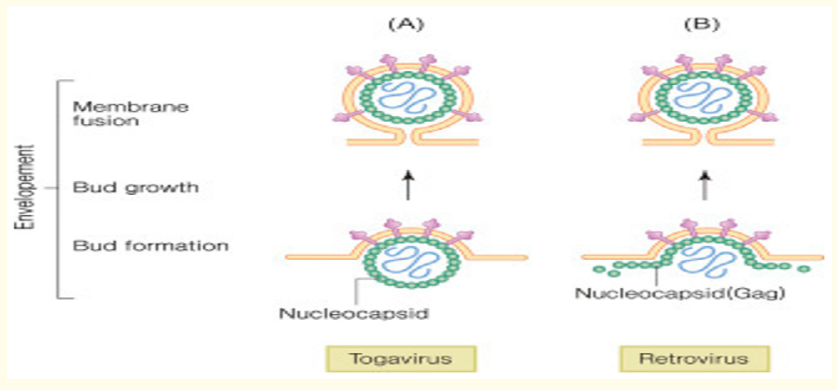

Figure 4: Relationship between capsid assembly and envelopment.

\section{Types of virus infection}

Virus infection can be classified as either "productive infection" or "nonproductive infection" depending on whether a progeny virus is created as shown in figure 5 .

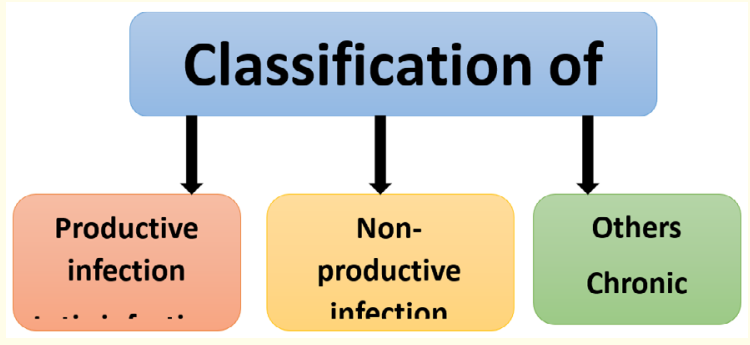

Figure 5: Types of viral infections.

\section{Persistent infection}

Persistent infection is not completely removed from the host's system but remains in the infected person's tissues or organs. The virus may remain silent or infect the host productively without causing major injury or death [15].

\section{Latent infection}

Latent infection under-take duplicate by the lytic cycle. Latency refers to the capacity of some viruses to remain hidden or resting inside the cell. This type of virus that can enter latency can cause acute infection before going resting. Chickenpox, for example, is an example of a contagious disease. A rash of blisters covers the skin. The sickness resolves 10 to 12 days after infection, and the virus stays dormant, remaining inactive for years within nerve-cell ganglia. The virus does not damage nerve cells or replicate during this time [16].

\section{Chronic infection}

Chronic infection is characterized by recurrent or persistent symptoms over a lengthy period. If the body is unable to clear the virus, some viral infections might become chronic e.g., HIV [17].

\section{Various approaches for the treatment of covid}

\section{SARS-CoV-2 life cycle and potential targets}

The life cycle of SARS-CoV-2 and potential antiviral medication targets for inhibition. This virus is called SARS-Cov-2, and it causes trouble swallowing, coughing, diarrhea, and, in extreme cases, breathing difficulties. More than 40,000 people have died in Mexico because of the SARS-Cov-2 pandemic [18]. because there is no viable treatment to halt the coronavirus from spreading over the world. SARS-CoV-2 specific effective medications have yet to be developed, and no specific drug for COVID-19 treatment has been licensed. Rapid evaluation of currently existing antiviral medications for COVID-19 patients, as well as the discovery of additional treatments, as a result, in this time of crisis, is extremely important. SARS-CoV-2 is a single-stranded RNA-enveloped virus that infects cells by connecting to the ACE2 receptor through the viral structural spike (S) protein [19].

\section{The current option bring tried for covid-19 treatment Synthetic/Semi-synthetic medications}

Various Pharmaceuticals existing drugs are repurposed for the therapeutic possibilities for COVID-19 management such as antiviral therapies, immune modulators, and supplementary therapy [20]. 


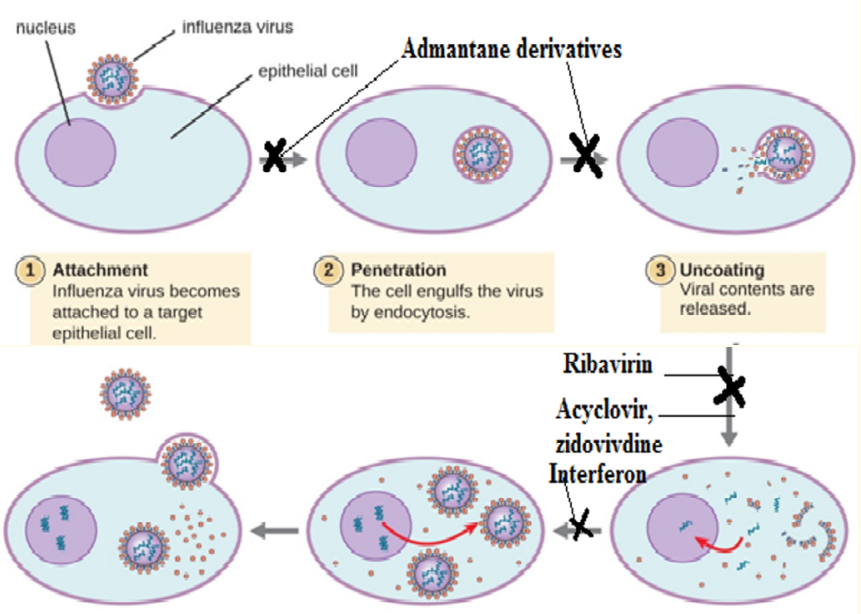

Figure 6: Different steps are act target for inhibition of virus infection.

\section{Antiviral drugs}

Because most COVID-19 patients have a moderate infection, antiviral medication may not be required in most cases. Our experience with influenza and severe acute respiratory syndrome (SARS) infections, on the other hand, suggests that starting antiviral medication early can help to shorten the disease's course. Despite the pressing need for antiviral medications, the bulk of these drugs are based on in vitro and extrapolated research [21].

\section{Remdesivir}

Remdesivir is a prodrug that is made up of nucleoside analogs. Remdesivir is an RNA-dependent polymerase inhibitor tested for efficacy in the treatment of SARS-CoV-2 infection and has demonstrated the most promising anti-viral therapeutic results [22], Remdesivir is an RNA-dependent polymerase inhibitor tested for efficacy in the treatment of SARS-CoV-2 infection and has demonstrated the most promising anti-viral therapeutic results. Antimicrobials activity against RNA viruses, such as Coronaviridae and Flaviviridae, were found during a screening approach for the agent. Due to its low EC50 and host polymerase selectivity against the Ebola virus, research and development of the agent showed promise during the peak of the Ebola virus outbreak [23]. Due to its broadspectrum, remdesivir is currently a viable possible therapy for COVID-19. Unlike other nucleoside analogs, remdesivir is a phosphoramide prodrug with broad-spectrum activity against a wide range of viruses, including Filoviridae, Paramyxoviridae, Pneumoviridae, and Orthocoronavirinae (SARS-CoV and MERS-CoV) [24].

\section{Lopinavir-ritonavir}

Lopinavir-ritonavir is an HIV protease inhibitor used in conjunction with other antiretroviral drugs to treat HIV-1 infection in adults, adolescents, and children over the age of two. When lopinavir-ritonavir was added to ribavirin, it lowered the likelihood of negative clinical outcomes and viral load in SARS patients. A prior study found that Kaletra ${ }^{\circledR}$, a specific combination of lopinavir and ritonavir, has antiviral activity against SARS-CoV in vitro and clinical trials [25].

\section{Ribavirin}

Ribavirin, a guanosine analog, prevents RNA and DNA viruses from replicating. In epidemics of SARS-CoV and MERS-CoV, ribavirin has been shown to have positive clinical effects. SARS-CoV has been effective against in-vitro, and inhibiting viral replication required high doses, essential high-dose, and combination therapy. The patients were given either intravenously or orally [26].

\section{Oseltamivir}

Oseltamivir binds to and inhibits the active site of the neuraminidase enzymes found on all influenza viruses in their active form inside the body. Because of oseltamivir's extremely selective action in influenza viruses, it is projected to be less effective in COVID-19. There was no effect on clinical outcomes in a Chinese observational study in which oseltamivir was given to $89.9 \%$ (out of 138) of patients [27].

\section{Adamantane derivatives}

Amantadine's replication was demonstrated to be inhibited by the first synthesized chemical. The structural properties of amantadine, rimantadine, and adamantane derivatives are similar and relate to their mechanism of action: blockage of the M2 channel, which transports $\mathrm{H}^{+}$ions (protons) into the inside of virions and starts the viral uncoating process. Amantadine was discovered to prevent the virus's penetration of the host cell, allowing the virus to remain susceptible to antibody inactivation for an extended period. With this antiviral "hit" in hand and clinical trials, several structural modifications alongside the "lipophilic, spheric cage hydrocarbon amine" are being investigated [28]. Amantadine and rimantadine are no longer the chemotherapeutic drugs of choice for treating in- 
fluenza. Anti-Influenza "adamantanes" are also launched and successfully used in clinical trials for the target to SARS co-V-2. Two potential treatments are amantadine and memantine. Low-cost, easily accessible drugs are a low-risk, low-cost, and cost-effective treatment option [29].

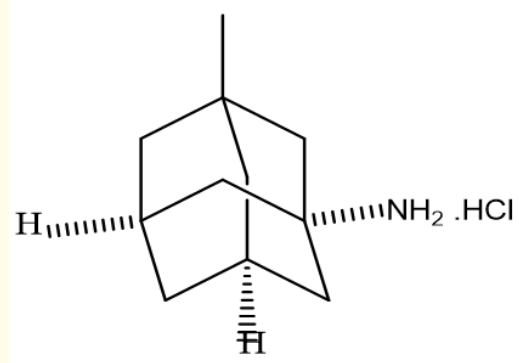

Amantadine

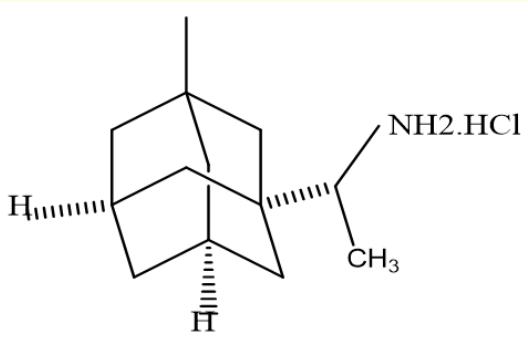

Rimantadine

Figure a

\section{Antimalarial drugs}

Chloroquine (CQ) and hydroxychloroquine (HCQ) can work in a variety of ways. It prevents viral particles from attaching to cellular receptors, disrupts early viral replication by changing the $\mathrm{pH}$ in endosomes, and prevents viral proteins from being modified after they have been translated. Furthermore, immune-modulatory effects from inhibiting interleukins (ILs) such IL-1beta and IL-6 help to reduce the inflammatory response to SARS-CoV-2 [30].

\section{Antibiotic drugs}

Azithromycin is a bacteriostatic antibiotic that's commonly used to treat a variety of Gram-positive infections. COVID-19 has been linked to secondary bacterial infection pneumonia in a few people. As a result, azithromycin is crucial in the treatment of bacterial pneumonia [31]. It has been discovered that, in addition to its antibacterial activity, it also possesses immunomodulatory and anti-inflammatory properties, suggesting that it may play a role in reducing the problems caused by respiratory viral infections such as SARS-CoV, MERS-CoV, and COVID-19 [32].

\section{Doxycycline}

Coronavirus has been shown to bind to the host's metalloproteases (MMPs) to ensure viral life. Antibiotics such as doxycycline have been shown to chelate zinc from MMPs. Doxycycline's chelating ability may aid in the prevention of COVID-19 infection. In France, nonhospitalized individuals with COVID-19 are being enrolled in phase III randomized, double-blind, placebo-controlled clinical trial to establish the safety and efficacy of doxycycline [33].

\section{Antiprotozoal drugs: Nitazoxanide}

It's a broad-spectrum antiparasitic drug that's been recommended for treating influenza and other viral respiratory infections [34]. Some researchers have suggested using nitazoxanide in combination with AZT and HCQ in COVID-19 [35]. However, no clinical trials have yet been conducted. Although in vitro evidence is promising, the paucity of clinical studies makes it difficult to recommend COVID-19 [36].

\section{Anti-parasitic drugs: Ivermectin}

Ivermectin suppresses the SARS-CoV-2, according to an in vitro investigation by Caly., et al. There are worries regarding drug therapeutic levels being reached in standard doses.

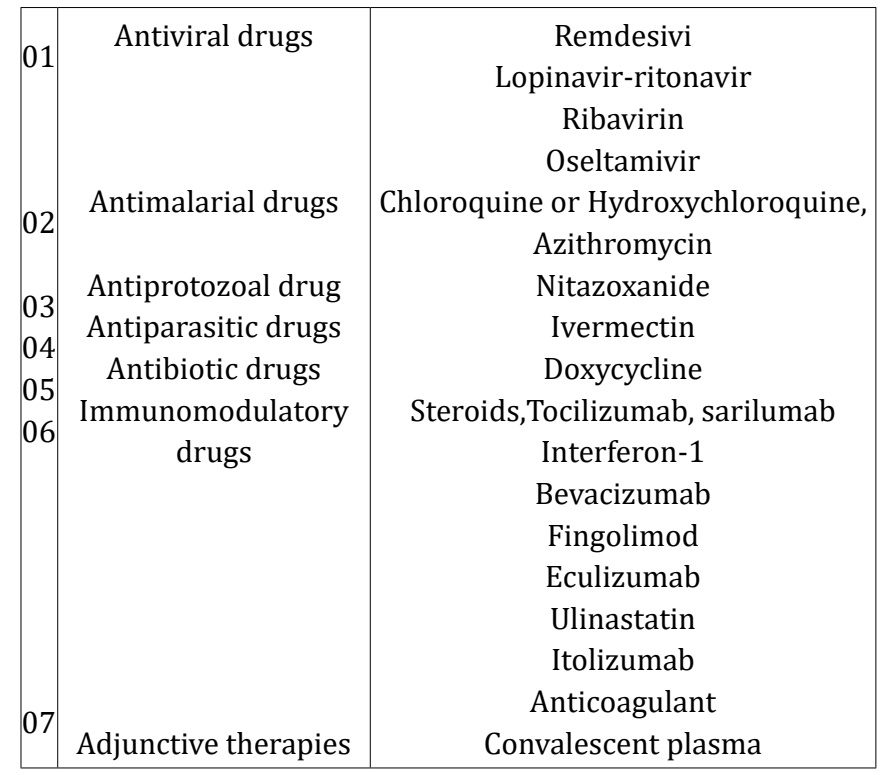

Table 1: Currently drugs under investigation for the treatment of COVID-19. 


\section{Biological approches}

\section{Convalescent plasma}

Convalescent plasma (CP) is a type of passive immunization in which prepared antibodies against an infectious agent are injected into a vulnerable host to prevent or treat infection [37]. Antibodies from COVID-19 patients' convalescent plasma can assist patients to achieve clinical remission and transient immunity [38]. This option comes with several drawbacks, the most significant of which are the variable humoral response, the lack of specific knowledge about the effective dose size or administration regimen, as well as the adverse effects. Through compassionate care initiatives, convalescent plasma has already been administered in critical COVID-19 patients, and as of April 2020, it will be used in all COVID-19 patients [39]. This medication is an FDA-approved "emergency experimental and novel medicine" in clinical studies. Some patients might be unable to participate in clinical trials because of the treatment's side effects. The substantial risk of transfusion-associated circulatory overload in this scenario limits or even prohibits the administration of plasma to ARDS or cardiac patients, underlining the necessity for well-documented RCTs on the subject [40].

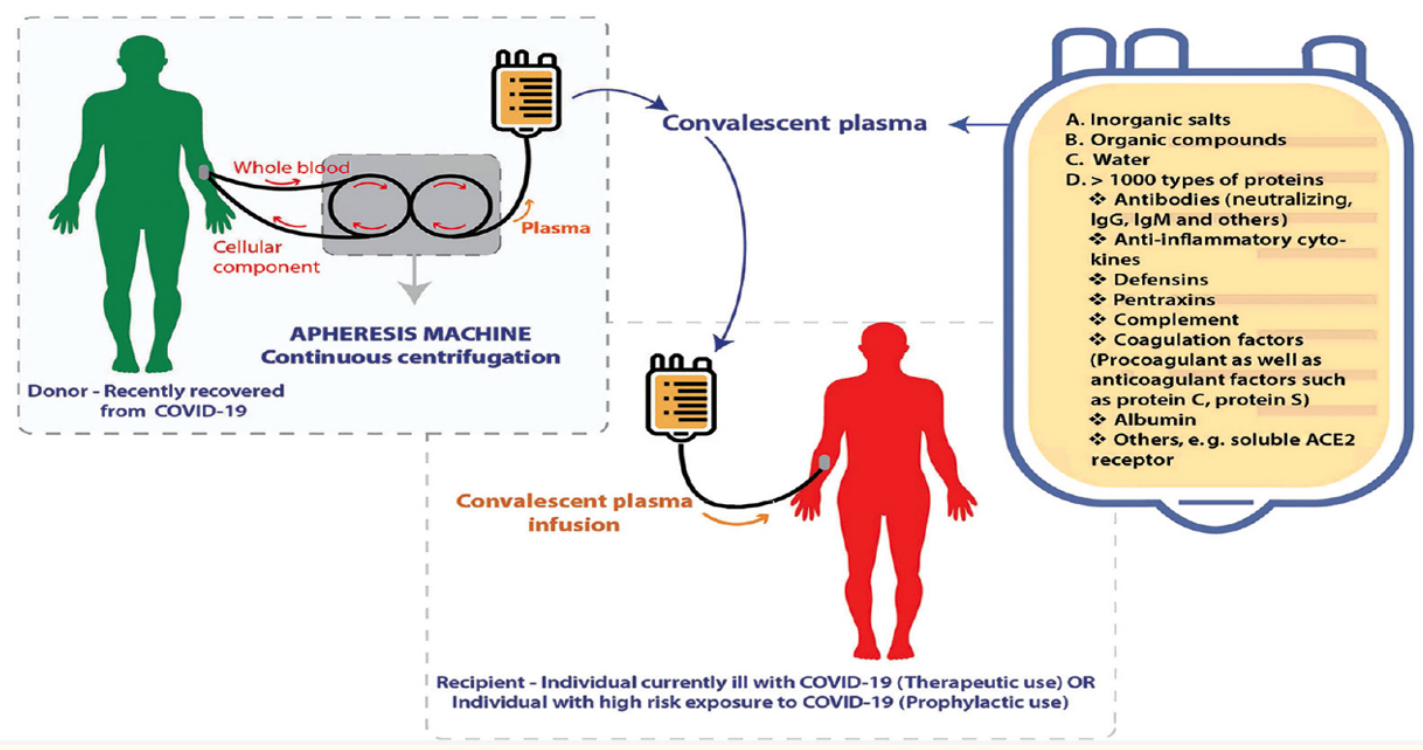

Figure 7: Convalescent plasma (CP) therapy is described in detail. The process of $\mathrm{CP}$ donation and its application in a patient with COVID-19 is depicted in the diagram.

\section{Vaccines}

Vaccine development is a difficult, time-consuming, and costly procedure. Multiple candidates are necessary, with multiple processes, pauses for inspections, and data analysis to eventually lead to a license production, due to high attrition rates [41]. A revolutionary experimental RNA-based vaccination (mRNA-1273) that employs part of the $\mathrm{S}$ protein genetic code is set to enter clinical trials as early as February 20b 20. It is being developed by Mod- erna Therapeutics, a pharmaceutical company that has previously worked on the SARS-CoV and MERS-CoV vaccines, allowing clinical development to bypass some animal testing based on previous research [42]. The lack of precise data on the immune response produced by SARS-CoV-2 and the substantial adverse effects identified in SARS-CoV and MERS vaccine candidates have hampered the development of a SARS-CoV-2 vaccine from the start [43]. Furthermore, lowering public confidence in the vaccination endeavor 
might be a significant roadblock to the creation of effective immunization programs. The rapid spread of the COVID-19 pandemic has encouraged the creation of technology platforms that are tailored to this novel pathogen and could be used to create a safe and effective vaccine. Over 100 SARS-CoV-2 potential vaccines are currently listed in the World Health Organization's draught landscape of COVID-19 vaccines. The majority of these potential vaccines are still in preclinical testing, but 19 have gone through clinical trials. 14 of these vaccines are based only on the S protein [44].

Vaccines are now in the pre-clinical stage of development for a large range of diseases. A new oral SARS-Cov-2 vaccination uses food-grade safe ingredients to target the $S$ protein. As a carrier, Saccharomyces cerevisiae. Given the difficulties associated with developing a vaccine against RNA viruses, experts believe that developing an effective vaccine for SARS-CoV-2 will be extremely difficult [45]. RNA viruses are notoriously difficult to create vaccines for; nonetheless, over 100 research groups, including biotech businesses and academic institutes, are now testing various techniques. While some of these vaccinations have begun human trials, others have not [46].

\section{Monoclonal antibody}

Tocilizumab is a monoclonal antibody that targets both soluble and membrane-bound interleukin- 6 receptors and is fully increased (IL-6R). In clinical care, it is used to treat adults with severe rheumatoid arthritis and children with systemic juvenile idiopathic arthritis [47]. Although its efficacy has recently been demonstrated in other systemic autoimmune and inflammatory conditions, such as giant cell arteritis, multicentric Castleman's disease [48], and most importantly, was approved by the FDA in 2017 for the treatment of Cytokine Release Syndrome in CAR T-cell cancer therapy, Increased IL-6 levels have been linked to increased mortality in COVID-19 [49]. Piquing interest in using tocilizumab as a COVID-19 treatment. Tocilizumab has been shown to improve oxygenation and reduce inflammatory biomarkers in COVID-19 patients who are hospitalized [49].

\section{Interferons}

Interferon was produced by the virally infected cells which protected the cells from further infection. A present the infections are synthesized by recombinant DNA technology. There are three major types of human interferon., alpha, beta, and gamma. the alpha interferon used as clinicallyInterferon-alpha-2a and alpha-2b are highly purified proteins with a molecular weight of 19000 which contain 165 amino acid residues. Alpha interferon either purified (Alfero- N) and recombinant (Intron-A, Roferon-A), is all available antiviral drugs [50].

\section{Conclusion}

This paper summarizes antiviral and supplementary drugs that could be used to treat SARS-CoV-2 infection, including rationales, docking, and modeling assessments, in vivo and in vitro findings, as well as results from novel exploratory drug protocols and clinical trials, during this emergency and crisis.

\section{Bibliography}

1. Andersen Paul. "Pathogenesis of lower respiratory tract infections due to Chlamydia, Mycoplasma, Legionella and viruses". Thorax 53.4 (1998): 302-307.

2. Jebril Nadia. "World Health Organization declared a pandemic public health menace: a systematic review of the coronavirus disease 2019 “COVID-19”'” (2020).

3. Santacroce Luigi., et al. "The human coronaviruses (HCoVs) and the molecular mechanisms of SARS-CoV-2 infection". Journal of Molecular Medicine 99.1 (2021): 93-106.

4. Arefi Maryam Feiz and Mohsen Poursadeqiyan. "A review of studies on the epidemic crisis of COVID-19 disease with a preventive approach". Work Preprint (2020): 1-13.

5. Holmes Emily A., et al. "Multidisciplinary research priorities for the COVID-19 pandemic: a call for action for mental health science". The Lancet Psychiatry 7.6 (2020): 547-560.

6. Banerjee Anwesha., et al. "Herpes simplex virus: the hostile guest that takes over your home". Frontiers in Microbiology 11 (2020): 733.

7. Baggen Jim., et al. "The life cycle of non-polio enteroviruses and how to target it". Nature Reviews Microbiology 16.6 (2018): 368-381.

8. Kelleni Mina T. "Nitazoxanide/azithromycin combination for COVID-19: A suggested new protocol for early management". Pharmacological Research 157 (2020): 104874.

9. Ryu Wang-Shick. "Virus life cycle". Molecular Virology of Human Pathogenic Viruses (2017): 31. 
10. Vyas SP and V Sihorkar. "Endogenous carriers and ligands in non-immunogenic site-specific drug delivery". Advanced Drug Delivery Reviews 43.2-3 (2000): 101-164.

11. Patel Leena N., et al. "Cell penetrating peptides: intracellular pathways and pharmaceutical perspectives". Pharmaceutical Research 24.11 (2007): 1977-1992.

12. Whittaker Gary R., et al. "Viral entry into the nucleus". Annual Review of Cell and Developmental Biology 16.1 (2000): 627651.

13. Kuznetsov Yurii G and Alexander McPherson. "Atomic force microscopy in imaging of viruses and virus-infected cells". Microbiology and Molecular Biology Reviews 75.2 (2011): 268285.

14. Morens David M and Anthony S Fauci. "Emerging pandemic diseases: how we got to COVID-19". Cell 182.5 (2020): 10771092.

15. Eccleston-Turner M and McArdle S. "Accountability, international law, and the World Health Organization; A need for reform?". Global Health 11.1 (2017): 27-39.

16. Boldogh I., et al. "Persistent viral infections". Medical Microbiology. $4^{\text {th }}$ edition (1996).

17. Roser Max., et al. "Coronavirus disease (COVID-19)-Statistics and research". Our World in data 4 (2020).

18. Yaqoob S., et al. "An overview of novel coronavirus sars-cov-2 spanning around the past, present, and future perspectives". Journal of Pure Applied Microbiology 14.1 (2020): 775-788.

19. Zhou Yadi., et al. "Artificial intelligence in COVID-19 drug repurposing". The Lancet Digital Health 2.12 (2020):e667-e676.

20. Yavuz Serap and Serhat Ünal. "Antiviral treatment of COVID-19". Turkish Journal of Medical Sciences 50.SI-1 (2020): 611-619.

21. Gordon Calvin J., et al. "Remdesivir is a direct-acting antiviral that inhibits RNA-dependent RNA polymerase from severe acute respiratory syndrome coronavirus 2 with high potency". Journal of Biological Chemistry 295.20 (2020): 6785-6797.

22. Siegel Dustin., et al. "Discovery and synthesis of a phosphoramidate prodrug of a pyrrolo [2, 1-f] [triazin-4-amino] adenine C-nucleoside (GS-5734) for the treatment of Ebola and emerging viruses" (2017): 1648-1661.
23. Sheahan Timothy P., et al. "Broad-spectrum antiviral GS-5734 inhibits both epidemic and zoonotic coronaviruses". Science Translational Medicine 9.396 (2017).

24. Chu CM., et al. "Role of lopinavir/ritonavir in the treatment of SARS: initial virological and clinical findings". Thorax 59.3 (2004): 252-256.

25. Stockman Lauren J., et al. "SARS: systematic review of treatment effects". PLoS Medicine 3.9 (2006): e343.

26. Srivatsan Padmanabhan M. "Potential dual therapeutic approach against SARS-CoV-2/COVID-19 with Nitazoxanide and Hydroxychloroquine" (2020).

27. Wanka Lukas Khalid Iqbal and Peter R Schreiner. "The lipophilic bullet hits the targets: medicinal chemistry of adamantane derivatives". Chemical Reviews 113.5 (2013): 3516-3604.

28. Popescu Razvan Andrei., et al. "Supportive Care: Low Cost, High Value". American Society of Clinical Oncology Educational Book 41 (2021): 240-250.

29. Devaux Christian A., et al. "New insights on the antiviral effects of chloroquine against coronavirus: what to expect for COVID-19?". International Journal of Antimicrobial Agents 55.5 (2020): 105938.

30. Pandey Abhjieet., et al. "Potential therapeutic targets for combating SARS-CoV-2: Drug repurposing, clinical trials and recent advancements". Life Sciences 256 (2020): 117883.

31. Sheahan Timothy P., et al. "Broad-spectrum antiviral GS-5734 inhibits both epidemic and zoonotic coronaviruses". Science Translational Medicine 9.396 (2017).

32. Dixit Subhal B., et al. "Current approaches to COVID-19: therapy and prevention". Indian Journal of Critical Care Medicine: Peer-Reviewed, Official Publication of Indian Society of Critical Care Medicine 24.9 (2020): 838.

33. Rossignol Jean-François. "Nitazoxanide, a new drug candidate for the treatment of Middle East respiratory syndrome coronavirus". Journal of Infection and Public Health 9.3 (2016): 227-230.

34. Kelleni Mina T. "Nitazoxanide/azithromycin combination for COVID-19: A suggested new protocol for early management". Pharmacological Research 157 (2020): 104874.

35. Srivatsan Padmanabhan M. "Potential dual therapeutic approach against SARS-CoV-2/COVID-19 with Nitazoxanide and Hydroxychloroquine" (2020). 
36. Bloch Evan M., et al. "Deployment of convalescent plasma for the prevention and treatment of COVID-19". The Journal of Clinical Investigation 130.6 (2020): 2757-2765.

37. Khaire Niranjan Shiwaji., et al. "Use of convalescent plasma for COVID-19 in India: A review and practical guidelines". The Indian Journal of Medical Research 153.1-2 (2021): 64.

38. Martins Filipe., et al. "Adverse effects of immune-checkpoint inhibitors: epidemiology, management and surveillance". Nature Reviews Clinical Oncology 16.9 (2019): 563-580.

39. Iacob Simona and Diana Gabriela Iacob. "SARS-coV-2 treatment approaches: numerous options, no certainty for a versatile virus". Frontiers in Pharmacology 11 (2020): 1224.

40. Gouglas Dimitrios., et al. "Estimating the cost of vaccine development against epidemic infectious diseases: a cost minimisation study". The Lancet Global Health 6.12 (2018): e1386e1396.

41. Jackson Lisa A., et al. "An mRNA vaccine against SARS-CoV2-preliminary report". New England Journal of Medicine 383.20 (2020): 1920-1931.

42. Flanagan Katie L., et al. "Progress and pitfalls in the quest for effective SARS-CoV-2 (COVID-19) vaccines". Frontiers in Immunology 11 (2020): 2410.

43. Silveira Marcelle Moura., et al. "DNA vaccines against COVID-19: Perspectives and challenges". Life Sciences (2020): 118919.

44. Jiang Shibo. "Don't rush to deploy COVID-19 vaccines and drugs without sufficient safety guarantees". Nature 579.7798 (2020): 321-322.

45. Shen Chenguang., et al. "Treatment of 5 critically ill patients with COVID-19 with convalescent plasma". JAMA 323.16 (2020): 1582-1589.

46. Fan Linzi., et al. "COVID-19 drug treatment in China". Current Pharmacology Reports 6.4 (2020): 146-154.

47. Galeotti Caroline., et al. "Sustained remission of multicentric Castleman disease in children treated with tocilizumab, an anti-interleukin-6 receptor antibody". Molecular Cancer Therapeutics 11.8 (2012): 1623-1626.

48. Zhou Fei., et al. "Clinical course and risk factors for mortality of adult inpatients with COVID-19 in Wuhan, China: a retrospective cohort study". The Lancet 395.10229 (2020): 1054-1062.
49. Price Christina C., et al. "Tocilizumab treatment for cytokine release syndrome in hospitalized patients with coronavirus disease 2019: survival and clinical outcomes". Chest 158.4 (2020): 1397-1408.

50. Pandya SN. "A Textbook of Medicinal Chemistry (Synthetic and Biochemical Approach)". SG Publisher, Varanasi 1 (2001): 107109.

\section{Assets from publication with us}

- Prompt Acknowledgement after receiving the article

- Thorough Double blinded peer review

- Rapid Publication

- Issue of Publication Certificate

- High visibility of your Published work

Website: www.actascientific.com/

Submit Article: www.actascientific.com/submission.php Email us: editor@actascientific.com

Contact us: +91 9182824667 